EPJ Web of Conferences 113, 08021 (2016)

DOI: $10.1051 /$ epjconf/201611308021

(C) Owned by the authors, published by EDP Sciences, 2016

\title{
Higgs particles interacting via a scalar Dark Matter field
}

\author{
Yajnavalkya Bhattacharya ${ }^{1,2, a}$ and Jurij Darewych ${ }^{2, b}$ \\ ${ }^{1}$ New Jersey Institute of Technology, Newark, NJ, USA \\ ${ }^{2}$ York University, Toronto, Canada
}

\begin{abstract}
We study a system of two Higgs particles, interacting via a scalar Dark Matter mediating field. The variational method in the Hamiltonian formalism of QFT is used to derive relativistic wave equations for the two-Higgs system, using a truncated Fockspace trial state. Approximate solutions of the two-body equations are used to examine the existence of Higgs bound states.
\end{abstract}

Dark matter particles (DM) of mass $m$ are described by a spinless, massive scalar feld $\phi,-$ interacting with the self-coupled Standard Model Higgs field $\chi$, with mass $\mu$. The Lagrangian density of this model is $(\hbar=c=1)$

$$
\begin{array}{r}
\mathcal{L}=\frac{1}{2} \partial^{\nu} \phi \partial_{\nu} \phi-\frac{1}{2} m^{2} \phi^{2}-\kappa \phi^{4}+\frac{1}{2} \partial^{\nu} \chi \partial_{\nu} \chi-\frac{1}{2} \mu^{2} \chi^{2}-\lambda v \chi^{3}-\frac{1}{4} \lambda \chi^{4} \\
-g_{1} \chi \phi^{2}-\eta_{1} \chi^{2} \phi^{2}-\eta_{2} \chi \phi^{3}-\eta_{3} \chi^{3} \phi-g_{2} \chi^{2} \phi
\end{array}
$$

where $\kappa, \lambda, g_{1}, g_{2}, v$ and $\eta_{j}(j=1,2,3)$ are coupling constants; $\lambda, \kappa, \eta_{j}$ being dimensionless, and $v, g_{i}$, $(i=1,2)$, having dimensions of mass. In canonical quantization the classical fields $\phi, \chi$ are promoted to operators.

In the Hamiltonian formalism of QFT, the equations to be solved are $\hat{P}^{\beta}|\Psi\rangle=Q^{\beta}|\Psi\rangle, \hat{P}^{\beta}=(\hat{H}, \hat{\mathbf{P}})$ and $Q^{\beta}=(E, \mathbf{Q})$ are the energy-momentum operator and corresponding eigenvalues. The $\beta=0$ (energy) component of the equation is generally impossible to solve. Approximate solutions can be obtained using the variational principle $\left\langle\delta \Psi_{\text {trial }}|\hat{H}-E| \Psi_{\text {trial }}\right\rangle_{t=0}=0$ where $\hat{H}$ is normal ordered, and $\left|\Psi_{\text {trial }}\right\rangle$ is a suitable trial state. Trial states are taken to be superpositions of channel Fock states. The simplest trial states that yield non-trivial results are

$$
\left|\psi_{\text {trial }}\right\rangle=\int \mathrm{d} \mathbf{p}_{\mathbf{1}} \mathrm{d} \mathbf{p}_{2} F_{1}\left(\mathbf{p}_{\mathbf{1}}, \mathbf{p}_{2}\right) h^{\dagger}\left(\mathbf{p}_{\mathbf{1}}\right) h^{\dagger}\left(\mathbf{p}_{2}\right)|0\rangle+\int \mathrm{d} \mathbf{p}_{\mathbf{1}} \mathrm{d} \mathbf{p}_{2} \mathrm{~d} \mathbf{p}_{\mathbf{3}} F_{2}\left(\mathbf{p}_{\mathbf{1}}, \mathbf{p}_{\mathbf{2}}, \mathbf{p}_{\mathbf{3}}\right) h^{\dagger}\left(\mathbf{p}_{\mathbf{1}}\right) h^{\dagger}\left(\mathbf{p}_{2}\right) d^{\dagger}\left(\mathbf{p}_{\mathbf{3}}\right)|0\rangle
$$

where $h$ denotes Higgs, $d$ Dark Matter operators that satisfy the usual commutation rules. $F_{i},(i=1,2)$ are variational channel wave functions.

For the case where $g_{1}=\eta_{j}=0$, the equations of motion, in the rest frame $\vec{Q}=0$, that follow from the variational principle are:

$$
F_{1}\left(\mathbf{q}_{1},-\mathbf{q}_{1}\right)\left[2 \omega\left(\mathbf{q}_{1}, \mu\right)-E\right]=-g_{2} \int \mathrm{d} \mathbf{p} \frac{F_{2}\left(-\mathbf{q}_{1}, \mathbf{q}_{\mathbf{1}}+\mathbf{p}, \mathbf{p}\right)}{(2 \pi)^{3 / 2} \sqrt{2 \omega\left(\mathbf{q}_{1}, \mu\right)} \sqrt{2 \omega(\mathbf{p}, m)} \sqrt{2 \omega\left(\mathbf{q}_{1}+\mathbf{p}, \mu\right)}},
$$

\footnotetext{
ae-mail: yajnaval@gmail.com

be-mail: darewych@yorku.ca
} 


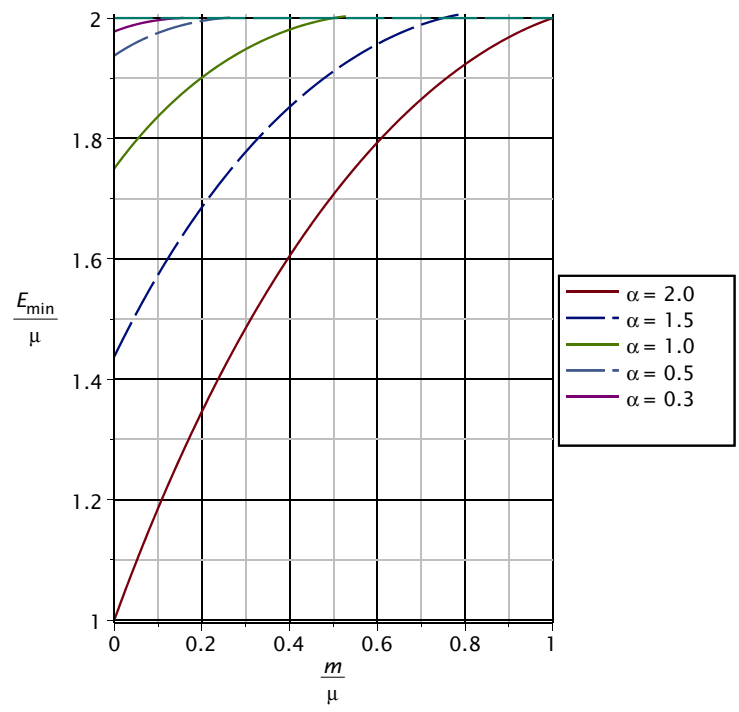

Figure 1. $E_{\min } / \mu$ as a function of $m / \mu$ for various values of $\alpha$. For a given value of $\alpha$, the two-Higgs ground state binding energy decreases with increasing $m / \mu$ from the Coulombic value, $\frac{1}{4} \mu \alpha^{2}$ at $m=0$, to zero at a critical value of $m$. The critical values of $m / \mu$, beyond which no two-Higgs bound states are possible correspond to points where the curves cross the line $E_{\min }=2 \mu$. These critical points occur where $m / \mu=\alpha /(2 Z)$, where $Z \lesssim 1$. Accurate numerical solutions of Equation (6) yield $Z=0.839908$.

$$
\begin{gathered}
F_{2}\left(\mathbf{q}_{1}, \mathbf{q}_{2}, \mathbf{q}_{1}+\mathbf{q}_{2}\right)\left[\omega\left(\mathbf{q}_{1}, \mu\right)+\omega\left(\mathbf{q}_{2}, \mu\right)+\omega\left(\mathbf{q}_{1}+\mathbf{q}_{2}, m\right)-E\right] \\
\quad=-g_{2} \frac{F_{1}\left(-\mathbf{q}_{2}, \mathbf{q}_{2}\right)}{(2 \pi)^{3 / 2} \sqrt{2 \omega\left(\mathbf{q}_{1}, \mu\right)} \sqrt{2 \omega\left(\mathbf{q}_{1}+\mathbf{q}_{2}, m\right)} \sqrt{2 \omega\left(-\mathbf{q}_{2}, \mu\right)}} .
\end{gathered}
$$

Exact, analytic solutions of the coupled, relativistic equations are not possible, so approximate variational-perturbative solutions will be considered. In the lowest order approximation, we set $\omega\left(\mathbf{q}_{1}, \mu\right)+\omega\left(\mathbf{q}_{2}, \mu\right) \simeq E$ in (4) whereupon equation (4) simplifies to

$$
F_{2}\left(\mathbf{q}_{1}, \mathbf{q}_{2}, \mathbf{q}_{1}+\mathbf{q}_{2}\right)\left[\omega\left(\mathbf{q}_{1}+\mathbf{q}_{\mathbf{2}}, m\right)\right]=-g_{2} \frac{F_{1}\left(-\mathbf{q}_{\mathbf{2}}, \mathbf{q}_{2}\right)}{\left.(2 \pi)^{3 / 2}\right) \sqrt{2 \omega\left(\mathbf{q}_{1}, \mu\right)} \sqrt{2 \omega\left(\mathbf{q}_{1}+\mathbf{q}_{2}, m\right)} \sqrt{2 \omega\left(-\mathbf{q}_{2}, \mu\right)}} .
$$

Thus in the rest frame, equation (3) becomes a single relativistic equation

$$
f(\mathbf{q})[2 \omega(\mathbf{q}, \mu)-E]=\alpha \mu^{2} \int \mathrm{d}^{3} \mathbf{p} \frac{f(\mathbf{p})}{\omega(\mathbf{q}, \mu) \omega^{2}(\mathbf{p}-\mathbf{q}, m) \omega(\mathbf{p}, \mu)} .
$$

where $f(\mathbf{q})=F_{1}(-\mathbf{q}, \mathbf{q})$, and $\alpha=g_{2}^{2} /\left(16 \pi^{2} \mu^{2}\right)$ is a dimensionless coupling constant.

Approximate variational solutions of (6) in the non-relativistic limit, for the ground state, are obtained using the trial state $f(\mathbf{p})=\frac{\omega(\mathbf{p}, \mu)}{\left(p^{2}+b^{2}\right)^{2}}$, where $\mathrm{b}$ is an adjustable parameter obtained by minimizing $E$. The results are given in Figure 1. 